TECHNICAL RESPONSE

WATER THERMODYNAMICS

\section{Response to Comment on "Maxima in the thermodynamic response and correlation functions of deeply supercooled water"}

\section{Kyung Hwan Kim, ${ }^{1}$ Alexander Späh, ${ }^{1}$ Harshad Pathak, ${ }^{1}$ Fivos Perakis, Daniel Mariedahl, ${ }^{1}$ Katrin Amann-Winkel, ${ }^{1}$ Jonas A. Sellberg, ${ }^{2}$ Jae Hyuk Lee, ${ }^{3}$ Sangsoo Kim, ${ }^{3}$ Jaehyun Park, ${ }^{3}$ Ki Hyun Nam, ${ }^{3}$ Tetsuo Katayama, ${ }^{4}$ Anders Nilsson ${ }^{1 *}$}

Caupin et al. have raised several issues regarding our recent paper on maxima in thermodynamic response and correlation functions in deeply supercooled water. We show that these issues can be addressed without affecting the conclusion of the paper.

〔 aupin et al. (1) question our application (2) of the Ornstein-Zernike (OZ) approach for noncritical divergence when the correlation length $\xi$ becomes small, of similar magnitude as the intermolecular distance, such that the division of $S(q)$ into normal and anomalous components strongly affects the resulting value of $\xi$. First, $\xi$ is not related to a specific distance in real space but is the damping factor in the asymptotic decay of the pair correlation (3). Second, we have followed exactly the same procedure in our determination of $\xi$ as earlier outlined by one of the authors of (I) [in (4)] for noncritical divergence based on OZ theory of $253 \mathrm{~K}$ water, using a division of $S(q)$ into anomalous and normal components.

Third, the argument against that approach given in reference 2 of (1) is valid for $298 \mathrm{~K}$ where the low- $q$ enhancement is extremely small, contrary to $280 \mathrm{~K}$ (see our determined structure factor variation in Fig. 1A where a detectable enhancement is clearly observed, which increases rapidly upon cooling). Figure 1B shows $\xi$ as a function of temperature, and indeed the error bars are large in the estimation of $\xi$ at high temperatures $(\sim 280 \mathrm{~K})$. At the lower temperatures, the enhancement of $S(q)$ at $q=0$ is approaching a factor of 2 in comparison to the minimum at $q=0.5 \AA^{-1}$, and the estimation of $\xi$ becomes increasingly independent of the choice of the normal component and the density $\rho$ used, resulting in small error bars as indicated in (2).

${ }^{1}$ Department of Physics, AlbaNova University Center. Stockholm University, SE-10691 Stockholm, Sweden. ${ }^{2}$ Biomedical and X-Ray Physics, Department of Applied Physics, AlbaNova University Center, KTH Royal Institute of Technology, SE-10691 Stockholm, Sweden. ${ }^{3}$ Pohang Accelerator Laboratory, Pohang, Gyeongbuk 37673, Republic of Korea. ${ }^{4}$ Japan Synchrotron Radiation Research Institute, Kouto 1-1-1, Sayo, Hyogo 679-5198, Japan.

*Corresponding author. Email: andersn@fysik.su.se and small at low temperatures.
Figure $4 \mathrm{~B}$ in (2) does not show clusters but is an illustration of fluctuating regions that vary in size as a function of temperature, as described in the caption. It is similar to the description from one of the authors of (1) [in (5)] as "transient hydrogen-bonded patches supported experimentally by the observation of small-angle x-ray scattering."

In (2), the compressibility $\kappa_{T}$ values are reported by using the extrapolated density $\rho$ below $239.74 \mathrm{~K}$, and the exact shape of $\kappa_{T}(T)$ depends on the choice of extrapolation to lower temperatures. We already tested the effect from the various extrapolations and have shown that it does not change the overall shape of the curve [sup- plementary text and figure S8 of (2)]. Caupin et al. propose to use a power law with $T_{\mathrm{s}}$ between 225 and $227 \mathrm{~K}$ that would yield a flatter temperature dependence instead of a maximum. There is a clear reason why $T_{\mathrm{S}}$ between 225 and $227 \mathrm{~K}$ should not be used in a power-law fit: $\rho$ would go significantly below the density of low-density amorphous ice at 228 and $229 \mathrm{~K}$ and would even become negative just above 225 and $227 \mathrm{~K}$, respectively, which would be unphysical. We note that two authors of (1) [in reference 6 of (1)] did not use what they propose in (1), but instead applied extrapolations of $\rho$ similar to those tested in (2).

The definition of the null hypothesis given in (1) [reproduced from (2)] is unusual. Although we did not assume any functional form for the test, specific functional forms were used in (I) (a parabola or a hyperbolic tangent function) even though the correct form is not known. In this case, the statistical hypothesis testing will relate to whether this specific functional form explains the experimental data well or not, and it is no longer related to the question regarding the presence or absence of a maximum; needless to say, there are an infinite number of other functional forms that do not exhibit a maximum. We also note that the appearance of a common maximum in all the eight plots, intensity of the small-angle $x$-ray scattering (SAXS), derivative of the first diffraction peak, $\xi$, and $\kappa_{T}$ for $\mathrm{H}_{2} \mathrm{O}$ and $\mathrm{D}_{2} \mathrm{O}$, shown in figures 2 and 3 of (2) adds further statistical significance to its existence.

The temperature has been estimated using the Knudsen evaporation model exactly in the same fashion as by two authors of (1) [in reference 6 of (1)]. The difference is that in reference 6 of (1), they determined the droplet diameter using Raman spectroscopy, but in (2) we could rely on the image from an optical microscope, as our droplet sizes are much larger.
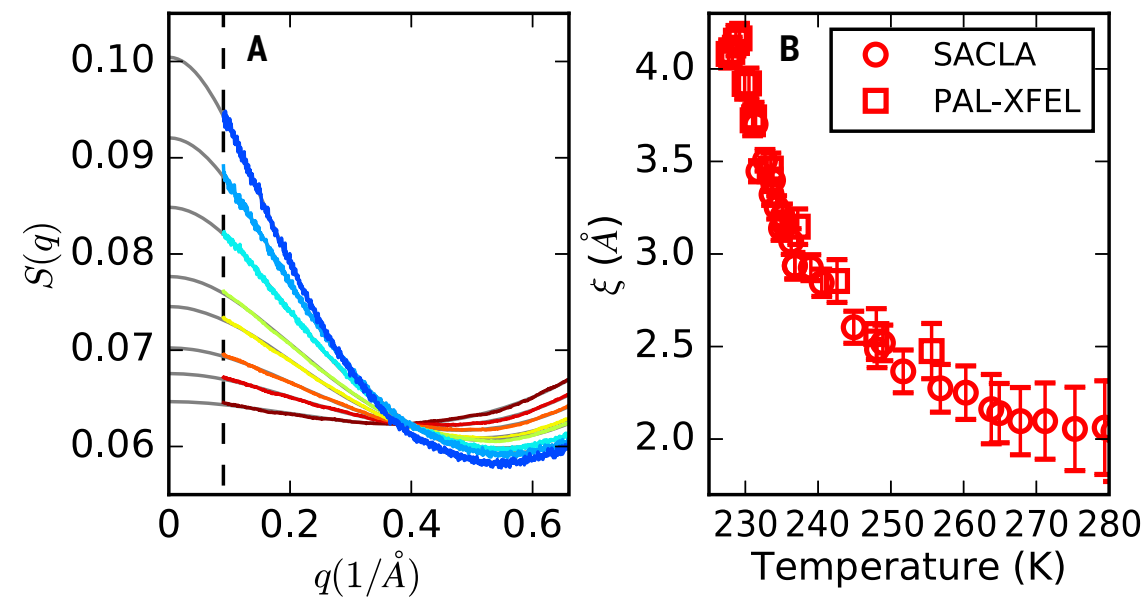

Fig. 1. OZ analysis of SAXS curves and correlation length. (A) Structure factor of $\mathrm{H}_{2} \mathrm{O}$ measured at SACLA from (2) for temperatures between $280 \mathrm{~K}$ (dark red) and $231 \mathrm{~K}$ (dark blue). Gray lines show the $\mathrm{OZ}$ fit using the $q$ range up to $0.65 \AA^{-1}$. (B) Correlation length obtained from the $\mathrm{OZ}$ fit for both the SACLA and PAL-XFEL data. The standard errors vary depending on the anomalous increase of the structure factor in $(A)$ and are relatively large at high temperatures 
It is true that metastability can occur for a hypothetical liquid-liquid phase transition, but the singularity point-free model would imply that the diverging temperature for the response functions would correspond to the spinodal of the transition where the metastability would be lifted exactly as stated by one of the authors of (1) [in (4)]. Because no phase transition is observed at the maxima of the response and correlation functions, there is no indication of a spinodal and therefore the critical point-free model is inconsistent with our experimental data. Furthermore, the change in the structure factor with temperature goes through a maximum, followed by a slower change and finally reaching a value close to that of low-density amorphous ice, indicating that a sharp but continuous transition has occurred.

Caupin et al. claim that we have in (2) overlooked their study [reference 8 of (1)], which cannot be the case when considering the dates: The journal receipt date of (2) is almost 1 month earlier than that of reference 8 of (1). We also note that reference 8 of (I) suggests that there are maxima in $\kappa_{T}$ at negative pressures, which is fully consistent with our findings - the existence of maxima of $\kappa_{T}$ at positive pressuresgiven in (2).

\section{REFERENCES AND NOTES}

1. F. Caupin et al., Science 360, eaat1634 (2018). 2. K. H. Kim et al., Science 358, 1589-1593 (2017).
3. A. Nilsson, C. Huang, L. G. M. Pettersson, J. Mol. Liq. 176, 2-16 (2012).

4. L. Bosio, J. Teixeira, H. E. Stanley, Phys. Rev. Lett. 46, 597-600 (1981).

5. M. C. Bellissent-Funel, J. Teixeira, L. Bosio, J. Dore, P. Chieux, Europhys. Lett. 2, 241-245 (1986).

\section{ACKNOWLEDGMENTS}

Supported by a European Research Council Advanced Grant under project no. 667205 and the Swedish National Research Council. The experiments were performed at beamline $\mathrm{NCl}$ of PAL-XFEL (proposal no. 2017-1st-CXI-006) funded by the Ministry of Science and ICT of Korea and at beamline BL3 of SACLA with the approval of the Japan Synchrotron Radiation Research Institute (proposal no. 2016A8015).

12 February 2018; accepted 13 April 2018

10.1126/science.aat1729 


\section{Science}

\section{Response to Comment on "Maxima in the thermodynamic response and correlation functions of deeply supercooled water"}

Kyung Hwan Kim, Alexander Späh, Harshad Pathak, Fivos Perakis, Daniel Mariedahl, Katrin Amann-Winkel, Jonas A. Sellberg, Jae Hyuk Lee, Sangsoo Kim, Jaehyun Park, Ki Hyun Nam, Tetsuo Katayama and Anders Nilsson

Science 360 (6390), eaat 1729.

DOI: $10.1126 /$ science.aat1729

ARTICLE TOOLS

http://science.sciencemag.org/content/360/6390/eaat1729

RELATED

http://science.sciencemag.org/content/sci/358/6370/1589.full

http://science.sciencemag.org/content/sci/360/6390/eaat1634.full

REFERENCES

This article cites 5 articles, 2 of which you can access for free http://science.sciencemag.org/content/360/6390/eaat1729\#BIBL

PERMISSIONS

http://www.sciencemag.org/help/reprints-and-permissions

Use of this article is subject to the Terms of Service

Science (print ISSN 0036-8075; online ISSN 1095-9203) is published by the American Association for the Advancement of Science, 1200 New York Avenue NW, Washington, DC 20005. 2017 () The Authors, some rights reserved; exclusive licensee American Association for the Advancement of Science. No claim to original U.S. Government Works. The title Science is a registered trademark of AAAS. 